\section{Data-Driven Design of a Dexterous Robotic Microsurgery System}

\author{
Frank L. Hammond III
}

Simon G. Talbot
Robert J. Wood
Robert D. Howe

School of Engineering and Applied Sciences Harvard University

\section{Background}

Critical microsurgery tasks such as small blood vessel anastomosis demand great manual dexterity and precision and are barely tenable by even the most practiced and skilled surgeons. Robotic micromanipulation devices are an obvious solution to this problem as they provide precise, repeatable motion at the sub-millimeter scale while filtering out physiological noise that limits the success of manual procedures. In order to be clinically effective and relevant, such robotic devices must provide significant improvements on the motion range, bandwidth, precision, and dexterity achievable with current manual microsurgery procedures [1].

This work comprises our first steps toward the empirical characterization of microsurgical workspaces necessary for the development of proper microsurgery robot performance specifications. In this study we record the motion of microsurgical tools during a simulated anastomosis procedure using an electromagnetic (EM) motion tracking system. The resulting data is used to establish microsurgery motion requirements which will inform the design of a robotic micromanipulation system that enables dexterous tissue handling and suturing on the sub-millimeter scale.

\section{Methods}

The microsurgical workspace required for the simulated small blood vessel anastomosis procedure was characterized by recording the motion of surgical tools using an (EM) motion tracking system (Ascension Tech Inc.). These surgical tools, which include forceps, a needle driver, and scissors, were fitted with small EM sensors whose linear position and angular orientation can be measured at maximum resolutions of $0.5 \mathrm{~mm}$ and $0.1^{\circ}$ respectively. EM sensors were mounted to the surgical tools using 3D-printed polymer sleeves designed to mitigate EM interference from proximity to ferromagnetic materials and to allow normal instrument usage without significant physical or visual impediment.

The motion tracking system was calibrated to determine the position of each surgical tool tip in relation to its mounted EM sensor. This was done by mechanically fixing the tool tip and rotating the tool about the fixed point while recording sensor positions. Using the empirical data, the kinematic transformation describing each tool tip position and orientation with respect its sensor coordinates was estimated using a Nelder-Mead simplex search for the transformation parameters that minimize the variance of tool tip positions.
An end-to-end anastomosis procedure was performed on lacrimal duct tubing, which simulates the scale and some mechanical properties of vascular tissue. The $1.0 \mathrm{~mm}$ diameter tubes were anastomosed with four interrupted 11-0 sutures. The procedure was performed multiple times with different lacrimal duct tube pairs to provide a larger, more reliable data set. All procedures were performed on a non-metallic workbench to reduce EM field distortions caused by the presence of ferromagnetic materials.

\section{Results}

The motion of the three microsurgery tools was recorded at a sampling rate of $50 \mathrm{~Hz}$. All motion data that did not involve lacrimal duct tube manipulation or suturing (e.g., tool retrieval motions) were removed from the data set by visual inspection, leaving behind only the clinically relevant portion of the workspace. Spectral analysis of tool tip motion yielded a linear motion bandwidth of $0-2.2 \mathrm{~Hz}$ and an angular motion bandwidth of $0-1.7 \mathrm{~Hz}$. Both fall below the $5-8 \mathrm{~Hz}$ human hand tremor bandwidth characterized in previous work [2]. Linear motion workspace dimensions were measured at $75.5 \mathrm{~mm}(x)$ by $184.0 \mathrm{~mm}$ ( $y$, along duct tubing axis) by $199.5 \mathrm{~mm}(z)$, and aggregate angular motion ranges about the $x$ (axial rotation), $y$ (elevation), and $z$ (lateral deviation) axes of the sensors were measured at $133.4^{\circ}, 29.0^{\circ}$, and $30.3^{\circ}$ respectively.

\section{Interpretation}

Analysis of the surgical tool motion data yielded several insights important to dexterous robotic microsurgery system design considerations including: (1) long surgical tools affect kinematic dexterity by decreasing angular motion range and increasing the angular resolution required to maintain tool tip precision, (2) EM sensors were swept through a volume roughly $85 \%$ larger than the tool tip volume due to tool length and manual dexterity limitations, and (3) the angular and linear motion bandwidths required for anastomosis do not seem to overlap with the human tremor bandwidth, making physiological noise filtering tractable. These insights, as well as quantitative data such as maximum tool tip velocity (11.86 $\mathrm{mm} / \mathrm{sec})$ and stroke length $(29.90 \mathrm{~mm})$, have informed the selection of linear translation stages (ATS100-100,100 $\mathrm{mm} / \mathrm{sec}$, Aerotech Inc.), the design of a high-precision, high bandwidth, screw-driven robotic wrist, and the selection of the motors, gearheads, encoders, and controllers (EPOS2, Maxon Precision Motors Inc.) required to drive the wrist.

Expanding this workspace characterization study to include other microsurgery fields such as otolarynogology and plastic surgery will provide a stronger empirical basis and result in richer performance specifications for the design of a versatile, clinically effective robotic microsurgery system.

\section{References}

[1] Taylor, R. et al., 1999, "A Steady Hand Robotic System for Microsurgical Augmentation," Int. Journal on Robotics Research, 18(12), pp. 1201-1210.

[2] Riviere, C., Ang, W., and Khosla, P., 2003, "Towards Active Tremor Canceling in Handheld Microsurgical Instruments," IEEE Trans. on Robotics and Automation, 19(5), pp. 793-800. 